\title{
Mapping of Microvascular Architecture in the Brain of an Alzheimer's Disease Mouse Model using MRI
}

\section{Suk-Ki Chang}

Hallym University Dongtan Sacred Heart Hospital

JeongYeong Kim

Kyung Hee University

\section{DongKyu Lee}

Ulsan National Institute of Science and Technology

Chang Hyun Yoo

Kyung Hee University

\section{Seokha Jin}

Ulsan National Institute of Science and Technology

\section{Hak Young Rhee}

Kyung Hee University Hospital at Gangdong

\section{Chang-Woo Ryu}

Kyung Hee University Hospital at Gangdong

Jong Kil Lee

Kyung Hee University

HyungJoon Cho

Ulsan National Institute of Science and Technology

Geon-Ho Jahng ( $\square$ ghjahng@gmail.com )

Kyung Hee University Hospital at Gangdong https://orcid.org/0000-0001-8881-1884

\section{Research}

Keywords: Alzheimer's disease, microvascular structure, MRI, contrast agent, animal study

Posted Date: June 2nd, 2020

DOI: https://doi.org/10.21203/rs.3.rs-29772/v1

License: (9) This work is licensed under a Creative Commons Attribution 4.0 International License.

Read Full License 
Version of Record: A version of this preprint was published at NMR in Biomedicine on February 15th, 2021. See the published version at https://doi.org/10.1002/nbm.4481. 


\section{Mapping of Microvascular Architecture in the Brain of an Alzheimer's Disease Mouse Model using MRI}

Suk-Ki Chang ${ }^{1}$, JeongYeong Kim² ${ }^{2}$, DongKyu Lee ${ }^{3}$, Chang Hyun Yoo $^{4}$, Seokha $\mathrm{Jin}^{3}$, Hak Young Rhee ${ }^{5}$, ChangWoo $\mathrm{Ryu}^{6}$, Jong Kil Lee ${ }^{7}$, HyungJoon $\mathrm{Cho}^{3}$, Geon-Ho Jahng ${ }^{6^{*}}$

The first two authors are co-first authors and contributed equally to this work.

${ }^{1}$ Hallym University medical center, Hwasung, South Korea

${ }^{2}$ Department of Physics, Undergraduate School, Kyung Hee University, \#26 Kyungheedae-ro, Dongdaemun-gu, Seoul 02447, Korea

${ }^{3}$ Department of Biomedical Engineering, Ulsan National Institute of Science and Technology, Ulsan, South Korea

${ }^{4}$ Department of Physics, Graduate School, Kyung Hee University, \#26 Kyungheedae-ro, Dongdaemun-gu, Seoul 02447, Korea

${ }^{5}$ Department of Neurology, Kyung Hee University Hospital at Gangdong, College of Medicine, Kyung Hee University, \#892 Dongnam-ro, Gangdong-gu, Seoul 05278 Republic of Korea

${ }^{6}$ Department of Radiology, Kyung Hee University Hospital at Gangdong, College of Medicine, Kyung Hee University,\#892 Dongnam-ro, Gangdong-gu, Seoul 05278 Republic of Korea

${ }^{7}$ Department of Pharmacy, College of Pharmacy, Kyung Hee University, \#26 Kyungheedae-ro, Dongdaemun-gu, Seoul 02447, Korea

Research Type: Original research

Running title: Microvascular MR imaging in AD Mouse brain

\section{*Contact information of the corresponding author:}

Geon-Ho Jahng, Ph.D, Professor of Radiology, Kyung Hee University Hospital at Gangdong, College of Medicine, Kyung Hee University, \#892 Dongnam-ro, Gangdong-Gu, Seoul 05278 Republic of Korea TEL: 822440 6187; FAX: 822440 6932; ghjahng@gmail.com 


\title{
Mapping of Microvascular Architecture in the Brain of an
}

\author{
Alzheimer's Disease Mouse Model using MRI
}

\begin{abstract}
Purpose: Growing evidence suggests that alterations of the cerebral microvasculature play a critical role in the pathogenesis of Alzheimer's disease (AD). The objective of this study was to characterize and evaluate the cerebral microvascular architecture in the $\mathrm{AD}$ transgenic $(\mathrm{Tg})$ mice model compared with non- $\mathrm{Tg}$ mice using brain microvascular indices obtained by MRI.
\end{abstract}

Methods: Seven non-Tg mice and ten 5xFAD Tg mice were scanned using a 7-T animal MRI system to measure the transverse relaxation rates of $\mathrm{R} 2$ and $\mathrm{R} 2 *$ before and after injection of the monocrystalline iron oxide nanoparticle contrast agent. After calculation of the relaxation rate difference of $\Delta \mathrm{R} 2 *$ and $\Delta \mathrm{R} 2$, the microvascular indices of the vessel size index (VSI), mean vessel diameter (mVD), mean vessel density (Q), mean vessel-weighted image (MvWI), and blood volume fraction (BVf) were mapped. The voxel-based analyses and regions-of-interest (ROIs)-based analyses were performed to compare the indices between the non$\mathrm{Tg}$ and $\mathrm{Tg}$ groups.

Results: The BVf, mVD, VSI, and MvWI were greater in the Tg group than in the non-Tg group based on voxel comparisons. Additionally, the ROIs-based analysis showed that $\Delta \mathrm{R} 2 *$, BVf, mVD, MvWI, and VSI were increased in the $\mathrm{Tg}$ group compared to the non- $\mathrm{Tg}$ group in several brain regions. Most ROIs defined by the mouse brain atlas were not significantly different between the two groups.

Conclusion: We found increased microvascular indices of VSI and $\mathrm{mVD}$ in the $\mathrm{Tg}$ mice, reflecting microvascular disruption in the brain, which may be related to damages of the neurovascular unit in AD caused by cerebral amyloid angiopathy.

Clinical Relevance / Application: To date, few studies have investigated the microvascular injuries of the AD brain using MRI. We suggest that investigation of cerebral microvasculature using a high-field MRI in AD 
would be worthwhile to elucidate the pathophysiology of cerebral amyloid angiopathy in this disease. Indeed, microvascular MRI can be a promising imaging tool for investigating early diagnosis and monitoring treatment in $\mathrm{AD}$.

Keywords: Alzheimer's disease; microvascular structure; MRI; contrast agent; animal study

\section{Introduction}

\section{Alzheimer's disease (AD) and its risk factors}

Alzheimer's disease (AD) is defined as a neurodegenerative disorder characterized by a gradual decline in memory and other cognitive functions and is neuropathologically associated with the accumulation of extracellular amyloid- $\beta$ peptide (A $\beta)$, intracellular neurofibrillary tangles, and hyper-phosphorylated microtubule-associated protein tau [1]. The microvascular structures in AD revealed by autopsy demonstrate decreased smooth muscle actin, resulting in an increase in wall thickness of the cerebral artery caused by accumulation of $\mathrm{A} \beta$ and/or the protein tau [2]. Hypertension, diabetes mellitus, dyslipidemia, and existing cardiovascular disease are the associated risk factors [1; 3] that contribute to the deterioration of the microvascular structure in the AD brain. Mechanisms of vascular pathology in AD include changes in vessel morphology, impairment of vascular reactivity, vascular stenosis, neurovascular uncoupling, and blood-brain barrier dysfunction.

\section{Cerebral amyloid angiopathy in the AD brain}

In $\mathrm{AD}$, cerebral amyloid angiopathy (CAA) is characterized by depositions of $\mathrm{A} \beta$ in the cortical and leptomeningeal vessel walls [4]. CAA causes a decrease in brain perfusion in AD due to compromised interstitial fluid drainage, resulting from the destruction of the perivascular spaces due to the microvascular accumulations of amyloid-beta. Senile plaques and CAA were observed in the brain of the AD model mouse with significant reduction in cerebral blood flow and decreased capillary density, with deleterious effects on 
cerebral microvasculature $[5 ; 6]$. Damage to the cerebral microvasculature is caused by abnormalities of the cerebrovascular walls, including smooth muscle cells, which show fibrinoid necrosis, as well as weakness of the vessel wall [7] and capillary occlusion,[8]. The formation of string vessels causes endothelial cell death, collapse of capillaries, and hypoperfusion [9]. Genetic risk factors for AD are associated with neurotoxicity of $\mathrm{A} \beta$ associated with CAA [10].

\section{Blood-brain barrier in the AD brain}

The blood-brain barrier (BBB) is a highly specialized endothelial cell membrane lining the cerebral microvessels, which is responsible for the generation and maintenance of chronic inflammation observed in $\mathrm{AD}$ by resident microglial cells and macrophages, along with circulating immune system cells, within the neurovascular unit (NVU). In AD, mechanisms of vascular pathology include changes in vessel morphology, impairment of vascular reactivity, vascular stenosis induced by endothelial fibrinogen deposition, neurovascular uncoupling and degeneration, and loss of vascular smooth muscle cells. Leukocyte trafficking, release of inflammatory mediators, and neutrophil extracellular traps also contribute to vascular dysfunction. As a result, the BBB dysfunction may eventually cause a microvascular injury. BBB dysfunction is caused by perivascular tau, and the associated neurotoxicity has been observed in transgenic mouse tauopathy models [11; 12]. In addition, $\mathrm{A} \beta$, CAA, and cerebral microbleeds (CMB) cause vessel wall injury by deposition of fibrinogen [12]. Vascular $A \beta$ is found on the arteries or arterioles, and on veins, with impaired drainage of interstitial fluid in animal models of AD [13]' [14]. The apolipoprotein E (ApoE) gene $\varepsilon 4$ allele is associated with increased plasma cholesterol and risk of neurovascular disease or atherosclerotic complications [15]. ApoE4 increases plasma protein, such as prothrombin in the microvessels, within the vascular basement membrane, resulting in breakdown and leakage of the blood-brain barrier due to shrinkage of endothelial cells [16]. Furthermore, ApoE4 causes proteolysis and cellular mitochondrial damage, which increases endothelial inflammation and facilitates the deposition of amyloid within the microvessels [17]. These factors may cause degradation of the $\mathrm{BBB}$; therefore, study of the microvasculature is important to increase comprehension of the pathologic mechanism of AD. 


\section{Microvascular imaging using MRI}

Microvascular MRI is a relatively new imaging method, which shows changes in signal intensity of susceptibility differences between blood vessels and surrounding tissues, based on the measurement of the changes of the transverse relaxation rate constants of $\Delta R_{2}$ and $\Delta R_{2} *$ before and after injection of an intravascular contrast agent (CA) $[18 ; 19]$. The CA increases the voxel relaxation rates by water diffusion between the intraand extra-vascular spaces and around the microvessels [19]. These changes in $\Delta \mathrm{R}_{2}$ and $\Delta \mathrm{R}_{2}^{*}$ in the microvasculature can be measured when the vessel is perpendicular to the main magnetic field. Therefore, the change depends on the microvessel radius and susceptibility difference $(\Delta \chi)$, which is the increase in intravascular magnetic susceptibility due to the CA [20]. To qualitatively and quantitatively measure the integrity of brain microvascular structures using MRI, the following microvascular indices were proposed: the vessel size index (VSI) [18], mean vessel diameter (mVD) [21], mean vessel density (Q) [19], mean vesselweighted image $\left(\mathrm{M}_{\mathrm{V}} \mathrm{WI}\right)$ [22], and blood volume fraction (BVf) [23]. Microvascular maps were applied in tumors [24-26], strokes [27], and dementia [28] to characterize vascular dysfunctions. However, few studies have been performed to investigate microvascular injuries in the AD-model mouse brain using MRI.

\section{Objective}

The objective of this study was to characterize and evaluate microvascular architectures based on brain microvascular indices obtained with a 7-T animal MRI system in Tg AD-model mice and the non-Tg mice using the monocrystalline iron oxide nanoparticle (MION) contrast agent.

\section{Materials and Methods}

\section{Animal preparation}

The animal experiment was approved by the Institutional Animal Care and Use Committee of Ulsan National Institute of Science and Technology (UNISTIACUC-16-26). The 5xFAD transgenic mouse, which is typically used in the AD-model, has 5 FAD mutations [APP K670N/M671L (Swedish) + I716V (Florida) + 
V717I (London) and PS1 M146L+ L286V][29]. Breeding progenitors of 5xFAD transgenic mice (stock number: 34840-Jax. strain name: B6SJL-Tg (APPSwFILon, PSEN1*M146L*L286V) 6799Vas/Mmjax) were purchased from The Jackson Laboratory (Bar Harbor, ME, USA). Breeding progenitors of B6/J (strain name: JAXTM C57BL/6J) and SJL/J mice (strain name: JAXTM SJL/6J) were purchased from The Charles River Laboratory (Yokohama, Kanagawa, Japan). To maintain the 5xFAD species, the breeding protocol of the 5xFAD mouse was guided by the Jackson Laboratory recommendation. In brief, B6SJLF1/J mice were produced by crossing a C57BL/6J female with SJL/J male. By mating the B6SJLF1/J with 5xFAD, B6SJLF1/J or 5xFAD mice were produced. In this study, 5xFAD and B6SJLF1/J sibling mice were used in the experiment and control group, respectively. All mice were housed in controlled conditions of a 12-h light/dark cycle with $21 \pm 2^{\circ} \mathrm{C}$ temperature and $50 \pm 10 \%$ humidity.

\section{MRI Acquisition}

In vivo MRI was performed with a mouse head surface coil on a Bruker 7-T Biospec small imaging scanner equipped with actively shielded gradients at a maximum strength of $76 \mathrm{G} / \mathrm{m}$ (Bruker Biospec GmbH, Ettlingen, Germany). Mice were initially anesthetized with $2.5 \%$ isoflurane in air. Experiments were carried out at a maintenance anesthesia level of $1.5 \%$.

Figure 1 shows the entire protocol of this study. First, a T1-weighted (T1W) fast low angle shot (FLASH) sequence was used to obtain anatomic information using the following parameters: repetition time $(\mathrm{TR})=800 \mathrm{~ms}$, echo time $(\mathrm{TE})=3.5 \mathrm{~ms}$, acquisition matrix $=256 \times 256$, number of slices $=10$, slice thickness $=1 \mathrm{~mm}$, flip angle $(\mathrm{FA})=50^{\circ}$, and number of averages $=3$. Second, to obtain brain anatomic information of the mice before and after injection of the contrast agent, two-dimensional (2D) T1W turbo spin-echo (TSE) images were acquired with the Rapid Acquisition with Refocused Echoes (RARE) sequence using the following parameters: $\mathrm{TR}=500 \mathrm{~ms}, \mathrm{TE}=7 \mathrm{~ms}$, turbo factor $=4$ (with centric encoding), acquisition matrix $=256 \mathrm{x} 256$, number of slices $=6$, slice thickness $=1.05 \mathrm{~mm}, \mathrm{FA}=90^{\circ}$, and number of averages $=6$. Third, to map the apparent diffusion coefficient (ADC), diffusion-weighted images (DWI) were acquired with a spin-echo echo planar imaging $(\mathrm{EPI})$ pulse sequence using the following parameters: $\mathrm{TR}=1000 \mathrm{~ms}, \mathrm{TE}=20 \mathrm{~ms}, 4 \mathrm{~b}$-values= $76.2165,618.8951,843.9312$, and $969.3098 \mathrm{~s} / \mathrm{mm}^{2}$, acquisition matrix $=128 \times 128$, number of slices $=6$, slice 
thickness $=0.7 \mathrm{~mm}, \mathrm{FA}=90^{\circ}$, and number of averages $=2$. The common imaging parameters were: field-ofview $(\mathrm{FOV})=16 \times 16 \mathrm{~mm}^{2}$. Fourth, to measure the $\mathrm{R} 2$ relaxation rates before and after injection of the contrast agent, 2D multi-slice multi-echo spin-echo (MESE) images were acquired using the following parameters: $\mathrm{TR}=$ $1300 \mathrm{~ms}$, TEs $=40,80,120$, and $160 \mathrm{~ms}$, acquisition matrix $=128 \times 128$, number of slices $=6$, slice thickness $=$ $0.7 \mathrm{~mm}, \mathrm{FA}=90^{\circ}$, and number of averages $=4$. Finally, to measure $\mathrm{R} 2 *$ relaxation rates before and after injection of the contrast agent, 2D multi-slice multi-echo gradient-echo (MEGE) images were acquired using the following parameters: $\mathrm{TR}=1500 \mathrm{~ms}$, TEs $=1.81,5.81,9.81,13.81,17.81,21.81,25.81,29.81,33.81$, and $37.81 \mathrm{~ms}$, number of slices $=6, \mathrm{FA}=60^{\circ}$, and number of averages $=2$. Slice thickness of $0.35 \mathrm{~mm}$ was used to reduce the signal distortion of gradient-echo imaging due to macroscopic B0 inhomogeneity. The MEGE acquisition was repeated twice to cover the same slice thickness corresponding to the MESE acquisition. A superparamagnetic MION was used as the contrast agent, with a dose of $200 \mu \mathrm{mol}$ of iron $/ \mathrm{kg}$.

\section{MR Imaging analysis}

\section{Pre-processing images}

Before we calculated $\mathrm{R} 2, \mathrm{R} 2 *, \mathrm{ADC}, \Delta \mathrm{R} 2, \Delta \mathrm{R} 2 *$, and microvascular indices, we pre-processed raw images with Statistical Parametric Mapping (SPM12) software (Wellcome Trust Centre for Neuroimaging, UCL Institute of Neurology, London, UK; www.fil.ion.ucl.ac.uk/spm) according to the following steps. First, the first spin-echo image before injection of contrast agent was used to co-register other raw images. All images were magnified by 10 for SPM software. Second, the first spin-echo image and other images were co-registered into C57B16 mouse brain template [30]. Finally, all images were spatially normalized into the template. After spatial normalization of all raw images into the mouse brain template, we mapped R2, R2*, and ADC maps.

\section{Mapping the $R 2$ and $R 2 *$ relaxation rates and $A D C$}

Before mapping the relaxation rates, the pre-processed images were de-noised with the Gaussian kernel size of $0.25 \times 0.25 \times 1 \mathrm{~mm}^{3}$. The changes in $\mathrm{R} 2$ and $\mathrm{R} 2 *$ relaxation rates before and after injection of the 
contrast agent should be calculated to map the microvascular indices. To calculate $\Delta R 2$, the acquired multi-slice MESE images were used with two different methods. First, the voxel-based R2 map was calculated by using a mono-exponential curve fitting method from signals collected before (preR2) or after (postR2) injection of the contrast agent, separately, and then voxel-based $\Delta \mathrm{R} 2$ can be obtained by subtracting preR2 from postR2 (postR2 - preR2). However, this method had unreliable results regarding $\Delta \mathrm{R} 2$ values in some voxels. Therefore, we used another method in which voxel-based $\Delta \mathrm{R} 2$ was directly calculated using the following equation [19].

$$
\Delta R_{2}=1 / T E \cdot \ln \left(S_{\text {post }} / S_{\text {pre }}\right)
$$

where $S_{\text {post }}$ and $S_{\text {pre }}$ are the signal intensities collected with $T E=80$ after and before injection of the contrast agent, respectively. Therefore, in this study, we ultimately used the second method to calculate $\Delta \mathrm{R} 2$ in each voxel.

To calculate $\Delta \mathrm{R} 2 *$, the acquired multi-slice MEGE images were used to map R2* before (preR2*) and after (postR2*) injection of the contrast agent. The voxel-based R2* was obtained by using a mono-exponential curve fitting method. Subsequently, voxel-based $\Delta \mathrm{R} 2 *$ was obtained by subtracting preR2* from postR2* (postR2*- preR2*). Finally, voxel-based ADC was calculated by using the mono-exponential curve fitting method with the multi-b-value DWIs.

\section{Mapping the microvascular indices}

The microvascular indices, VSI, BVf, mVD, Q, and MvWI, were mapped with the equations explained in this section using the parameter maps of $\Delta \mathrm{R} 2, \Delta \mathrm{R} 2^{*}$, and/or ADC. First, the blood volume fraction (BVf, \%) was obtained by using T2*-weighted sequence; therefore, BVf in a voxel is directly proportional to $\Delta \mathrm{R}_{2} *$ [23]. The $\mathrm{BVf}$ in a voxel is directly related to the changes in $\mathrm{R} 2 *$ between the before and after injection conditions using a paramagnetic contrast agent in the microvasculature. In assuming that a voxel has thousands of microvessels and occupies a small blood fraction of the voxel volume [19], the total BVf in a voxel was calculated with the following equation:

$$
B V f=\frac{3}{4 \pi} \cdot \gamma \cdot \Delta \chi \cdot B_{0} \cdot \Delta R_{2}^{*} \cdot 100(\%)
$$

where $\gamma$ is the gyromagnetic ratio $\left(\mathrm{Hz} \cdot \mathrm{T}^{-1}\right), \mathrm{B}_{0}$ is the intensity of the main magnetic field $(\mathrm{T})$, and $\Delta \chi$ (in cgs 
units) is the increase in intravascular magnetic susceptibility due to the concentration and susceptibility of the contrast agent. Note that $\Delta \chi$ depends on the type and on the concentration of the contrast agent. We used 3.6E05 as the $\Delta \chi$ value.

Second, the vessel size index (VSI, expressed in $\mu \mathrm{m}$ ) is a characteristic of the distribution of the vessel radii [18]. VSI was calculated by using $\Delta \mathrm{R}_{2}, \Delta \mathrm{R}_{2}{ }^{*}, \Delta \chi$, and ADC [18]. In assuming that the microvasculature has a uniform distribution of randomly oriented straight cylinders, as well as the small BVf $(<<1)$ and large echo times $\left(\mathrm{TE}>>1 /\left(2 \pi \cdot \gamma \cdot \Delta \chi \cdot B_{0}\right)\right.$, VSI in a voxel was computed by considering the water diffusion and the concentration of the contrast agent as:

$$
V S I=0.424 \cdot\left(\frac{A D C}{\gamma \cdot \Delta \chi \cdot B_{0}}\right)^{\frac{1}{2}} \cdot\left(\frac{\Delta R_{2}^{*}}{\Delta R_{2}}\right)^{\frac{3}{2}}
$$

where $A D C$ is the water diffusion rate $\left(\mu \mathrm{m}^{2} / \mathrm{s}\right)$.

Third, the mean vessel diameter (mVD) was introduced as the ratio of the changes in relaxation rates [21]. The mVD provides an estimate of microvascular changes, is sensitive to the average vessel size in the voxel, and is valid for small coefficients of variation in vessel size distribution [21]. The mVD in a voxel was calculated by the ratio of the changes in transverse relaxation rates:

$$
m V D=\frac{\Delta R_{2}^{*}}{\Delta R_{2}}
$$

Fourth, the quantity of mean vessel density ( $Q$, expressed in $\mathrm{s}^{-1 / 3}$ ) was introduced to minimize the dependence of the CA concentration, which is sensitive to intrinsic tissue properties [19] [31]. Therefore, it can be useful for an in vivo study, as the CA concentration may pose as a challenge due to this sensitivity to intrinsic tissue properties, whereas the estimation of vessel size requires the intravascular CA concentration [32]. Mean vessel density in a voxel was calculated with the following equation:

$$
Q=\frac{\Delta R_{2}}{\left(\Delta R_{2}^{*}\right)^{\frac{2}{3}}}
$$

Finally, the microvessel-weighted image $\left(\mathrm{M}_{\vee} \mathrm{WI}\right.$, expressed in $\left.\mathrm{s}^{-2}\right)$ was proposed to map small blood vessels [22] using the following equation.

$$
M V W I=\Delta R_{2}^{*} \cdot \Delta R_{2}
$$




\section{Statistical analysis}

\section{Voxel-based analysis}

All maps, which were the microvascular indices, $\Delta \mathrm{R} 2, \Delta \mathrm{R} 2^{*}$, and $\mathrm{ADC}$, were smoothed with $8 \times 8 \times 8$ $\mathrm{mm}^{3}$ full width of half maximum of Gaussian kernel. To compare all maps between the non- $\mathrm{Tg}$ and $\mathrm{Tg}$ groups, the voxel-based independent t-test was used. The month was used as a covariate. The significance level was $\mathrm{p}=0.001$ without correcting for the multiple comparisons, and with the threshold of 30 contiguous voxels. The voxel-based analyses were performed to select brain areas to obtain the microvascular index values for the regions-of-interest (ROIs)-based analyses.

\section{ROI-based analysis}

Two methods were used to define ROIs. First, the results of the voxel-based analyses were used to define ROIs. The locations of the coordinates of the significant difference of microvascular indices between the non- $\mathrm{Tg}$ and $\mathrm{Tg}$ groups were selected as the center with the sphere radius $=5 \mathrm{~mm}$. Note that we enlarged the images to 10 times the size of the original image. We obtained microvascular index values from the ROIs using the Marsbar tool (http://marsbar.sourceforge.net/).

Second, ROIs were defined with the mouse brain atlas at the right and left somatosensory cortexes, CA1, hippocampi, dentate gyri, and thalami using the wfu_pickatlas tool (http://fmri.wfubmc.edu/software/pickatlas) and the Franklin and Paxinos atlas [33]. Figure 2 shows the ROIs defined by the two methods. To compare the index values between the non-Tg and Tg mice, the Mann-Whitney test was used with the significance level of $\mathrm{p}=0.05$ in the ROI analyses. MedCalc statistical software (http://www.medcalc.org/, Ostend, Belgium) was used to analyze the data.

\section{Histology}

Histology was performed in two different brain areas in the $\mathrm{Tg}$ and non-Tg mice. First, for vascular imaging with confocal microcopy at the left somatosensory cortexes, 0.1\% TRITC-dextran (Sigma, 65 85 kDa) 
with 5\% gelatin solution was perfused through the heart. Subsequently, the brain was extracted and fixed with $4 \%$ PFA solution for approximately one day. For the frozen section, the brain was embedded into the Tissue-Tek OCT compound and sliced to a thickness of $50 \mu \mathrm{m}$. The slides were scanned with confocal microscopy (OLYMPUS, FV-1000, x10 magnification). One 5XFAD mouse and one wild type mouse were used to analyze vessel histology at the left somatosensory cortexes.

Second, the other six-month-old male mice, which were four 5XFAD mice and three wild-type mice, were anesthetized with intraperitoneal injection of $2.5 \%$ Avertin (2, 2, 2-tribromoethanol), and cardiac perfused immediately with PBS followed by $4 \%$ paraformaldehyde in PBS. Areas of the brains surrounding the thalamus and hippocampus were excised and post-fixed overnight in $4 \%$ paraformaldehyde at $4{ }^{\circ} \mathrm{C}$ and incubated in $30 \%$ sucrose at $4{ }^{\circ} \mathrm{C}$ until equilibration. The brains were embedded into OCT compound blocks at $-80{ }^{\circ} \mathrm{C}$. Sequential 30- $\mu \mathrm{m}$ coronal sections were obtained with a cryostat (CM30 50S; Leica) and free-floating brain sections were used to visualize brain vessels. Sections were incubated with Dylight 594-conjugated L. esculentum lectin (1:100, Vector Laboratories, DL-1177) and the samples were analyzed with a BX51 microscope (Olympus). The quantification of vessel areas in the hippocampus and thalamus was performed using Image $\mathbf{J}$ software.

\section{Results}

Figure 3 shows the representative maps of $\Delta \mathrm{R} 2$ and $\Delta \mathrm{R} 2 *$ relaxation rates, $\mathrm{ADC}$, and microvascular indices obtained from the non- $\operatorname{Tg}$ (10 months) and $\operatorname{Tg}(2$ months) mice. All maps were calculated after applying the Gaussian kernel of $0.25 \times 0.25 \times 1 \mathrm{~mm}^{3}$ to reduce the noise. The window level for each microvascular index was the same for non- $\mathrm{Tg}$ and $\mathrm{Tg}$ mice.

\section{Voxel-based analysis}

Figure 4 shows the resulting statistical maps of the voxel-based comparisons of the microvascular indices between the two groups. Note that there were no areas shown with large microvascular index values in the non- $\mathrm{Tg}$ group compared with the Tg groups. Table 1 summarizes the results of the voxel-based comparisons 
for each microvascular index between the two groups. $\Delta \mathrm{R} 2$ was greater in the $\mathrm{Tg}$ group than in the non- $\mathrm{Tg}$ group at the somatosensory cortex. $\Delta \mathrm{R} 2 *$ was also greater in the $\mathrm{Tg}$ group than in the non-Tg group at the somatosensory cortex, cerebral cortex, and motor cortex. BVf had a similar result as the $\Delta \mathrm{R} 2 *$. The mVD, VSI, and MvWI were also greater in the Tg group than in the non-Tg group. The ADC and mean vessel density $\mathrm{Q}$ were not significantly different between the two groups.

\section{ROI-based analysis}

Table 2 lists the results of the ROI-based analysis of the microvascular indices to compare those between the non-Tg and Tg groups. $\Delta \mathrm{R} 2 *$ and BVf were significantly different between the two groups at ROI 1 to 5 and SOM but were not for other ROIs. The mVD and VSI were significantly different between the two groups at ROI 1 to 3 but were not different for other ROIs. MvWI was significantly different between the two groups at ROI 2 and ROI 3 to 5 but was not different for other ROIs. $\Delta$ R2 and Q were not significantly different between the two groups for all ROIs. Most ROIs defined by the result of the voxel-based analysis were significantly different between the two groups; however, most ROIs defined by the mouse brain atlas were not significantly different. Figure 5 shows the representative results of the comparisons of the microvascular indices obtained from the right somatosensory cortex (a.ROI2) and the hippocampus (b.HP) listed in Table 2. Microvascular indices were significantly increased in the Tg mouse compared with the non-Tg mouse in the right somatosensory cortex, but not in the hippocampus.

\section{Tissue histology}

Figure 6a shows vascular structures of the left somatosensory cortexes for a non-Tg mouse (left) and a 5xFAD Tg mouse (right). The microvascular vessels were destroyed in the Tg mouse, and the relatively large vessels are enlarged. Figure $\mathbf{6 b}$ shows the results of histology around the thalamus and hippocampus. The microvascular structures of the $\mathrm{Tg}$ mouse were similar to those of the non-Tg mouse. The dextran-positive area was slightly increased in the $\mathrm{Tg}$ mouse without statistical significance. This result might be associated with enlargement of vessel diameter. 


\section{Discussion}

$\mathrm{CAA}$ is responsible for the pathogenesis of $\mathrm{AD}$, since $\mathrm{A} B$ deposited in arteries or arterioles (less frequently in capillaries and veins) can cause microvascular degeneration. Some theories suggest that cerebrovascular dysfunction precedes cognitive decline, and the onset of neurodegenerative changes in $\mathrm{AD}$ indicate that cerebral hypoperfusion impairs the clearance of $A ß$ from the brain, which is normally performed by the cells in the neurovascular unit. Therefore, $A ß$ accumulates in blood vessels and in brain parenchyma. Comparison of $\mathrm{Tg}$ mouse models for $\mathrm{AD}$ to controls provides an excellent opportunity to study the impact of microvascular dysfunction on the progression of $A ß$ deposition [34]. Therefore, the aim of this study was to noninvasively investigate microvascular architectures in the brain of the Tg AD-model using MRI. We obtained two important results. First, the microstructure in the AD-model with $\mathrm{Tg}$ mice was damaged in several regions of the brain. Second, the microvascular indices of $\mathrm{mVD}$, VSI, and $\Delta \mathrm{R} 2 *$ were sensitive to monitoring of the microvascular changes in the $\mathrm{AD}$-model of the $\mathrm{Tg}$ mouse, while $\Delta \mathrm{R} 2$ and $\mathrm{Q}$ were less sensitive. We discussed these findings in this session. Our findings may guide future attempts at applying microvascular imaging to the $\mathrm{AD}$ human brain. We suggest that the investigation of cerebral microvasculature using a high-field MRI in $\mathrm{AD}$ may be worthwhile to elucidate the pathophysiology of CAA in AD.

\section{Microstructures are damaged in the AD-model Tg mouse.}

Our results showed that the microvascular damages in the $\mathrm{Tg}$ mouse were spatially localized at the cortical areas, such as the primary and secondary somatosensory cortex, supported by the histology as seen in Figure 6. A previous animal study also showed that the relative cerebral blood volume in the AD mouse $\left(\mathrm{APP}_{\mathrm{swe}} / \mathrm{PS} 1_{\mathrm{dEg}}\right)$ model decreased mostly in the primary and secondary somatosensory areas [28]. However, other previous animal studies did not observe a decrease in microvascularity in the thalamus and hippocampus 
$[15 ; 35]$. Previous studies showed that 5xFAD transgenic mice have many of the behavioral and neuropathological traits of $\mathrm{AD}$, such as brain parenchymal $\mathrm{A} \beta$ accumulation, and manifest similarities to $\mathrm{AD}$ in human brain models, including abnormalities in the cerebral cortical microvasculture, and early impairment in cerebral blood flow and cerebrovascular autoregulation $[15 ; 28]$. In general, cerebral microvascular pathology causes senile cognitive dysfunction and neurodegeneration in the AD-model mouse [11]. The vascular amyloid$\beta$ accumulation could impair cell function leading to vascular function impairment.

A previous post-mortem study of the human $\mathrm{AD}$ brain showed vascular pathology factors, including Endothelin 1 (EDN1), vascular endothelial growth factor (VEGF), and von Willebrand factor (VWF) [35]. Recent human studies also showed evidence for impaired vasculature, a causative factor in AD pathology progression $[3 ; 8]$. In $\mathrm{AD}$, vasoconstriction is caused by deposition of amyloid- $\beta$ peptides, which resulted in the reduction of cerebral blood flow and impairment of both functional hyperemia and cerebral autoregulation, and is significantly correlated with the severity of small vessel disease-associated WM hypoperfusion, or cerebral amyloid angiopathy, in the neocortex. Vascular dysfunction in the brain has long been recognized as a contributing factor to the development of various brain diseases, including stroke, vascular dementia, and AD [36]. These topics have been studied in vivo, which include anatomy of the blood supply, tortuous vessels, venous collagenosis, string vessels (capillary remnants), decreased vascular density, and microembolic brain injury. Other vascular risk factors are basement membrane (BM) thickening, cerebral hypoperfusion, and extravasation of emboli. Microembolic injury of the cerebral vessels leads to cognitive decline, as AD and vascular cognitive impairment follow leukoaraiosis, which is a white matter (WM) degeneration characterized by gliosis, demyelination, and capillary degeneration, as well as endothelial dysfunction [9; 15; 37].

\section{$\Delta R 2 *, m V D$, and VSI are sensitive to evaluation of changes in vascular structure in AD.}

Our results showed that $\Delta \mathrm{R} 2 *, \mathrm{BVf}, \mathrm{mVD}, \mathrm{VSI}$, and MvWI sensitively detected the difference between the two groups at some ROIs defined by the voxel-based analysis; however, $\Delta \mathrm{R} 2$ and $\mathrm{Q}$ did not. Furthermore, microvascular indices were not significantly different between the two groups at most ROIs defined by the mouse brain atlas. Cerebral microvessels, which include capillaries, small arterioles, and venules, exhibit multiple dynamic responses to morphological variations of the microvascular network together with neighboring 
neurons. There is no way to image the microvascular network directly in the scale of micrometers. Therefore, parameters reflecting properties of the local microvascular network in the imaging scale are used in this study.

$\Delta \mathrm{R}_{2}$ * index was obtained with a multi-echo gradient-echo sequence before and after injecting the contrast agent for susceptibility weighting of all vessel sizes, increasing the difference between blood and the surrounding tissue. In general $\Delta \mathrm{R}_{2} *$ reflects total blood volume, including both macrovessels and microvessels; however, $\Delta \mathrm{R}_{2}$ is a measure of primarily microvascular blood volume in capillaries and venules $[18 ; 38 ; 39]$. Therefore, our results demonstrated the increase of vessel diameter, which is represented by mVD. The BVf is also proportional to $\Delta \mathrm{R}_{2}{ }^{*}$. Therefore, $\Delta \mathrm{R}_{2} *$ index may serve as a sensitive reliable marker in neurovascular pathology, to improve evaluation of microvascular architecture. A previous study showed that the $\Delta \mathrm{R}_{2} *$ index was affected by neurogenesis, such as the migration of neuroblasts, vascular remodeling, and increased vessel density, as well as dendritic plasticity, characterized by T2* shortening [39].

The mVD and VSI measure the mean vessel diameter qualitatively and quantitatively, respectively. VSI is dependent on blood flow and oxygenation of the tissue, but less dependent on the geometry of blood vessels. VSI was considered as a noninvasive index to describe the pathologic changes of vessels in quantitative terms. It is a mathematically expressed parameter, more specifically, an averaged radius of all the microvessel sizes contained within an imaging voxel, and is understood as a mean vessel radius averaged over the capillary population with the weight of its volumetric fraction. Therefore, it is valuable to monitor the dilation and contraction of microvessels. The calculation of the quantitative VSI is based on the combined estimations of accurate $\mathrm{CBV}$, ADC, and $\mathrm{Q}[38 ; 40]$. The $\operatorname{mVD}\left(\Delta \mathrm{R}_{2} * / \Delta \mathrm{R}_{2}\right)$ probes the distribution of submillimeter caliber microvessels induced by a superparamagnetic contrast agent, and is a direct measure of the average vessel radius. However, intrinsic venous blood paramagnetism may result in systematic overestimation of vessel size, although it is less problematic for contrast agents with high susceptibility [20]. In this study, we found increased mVD and VSI in the Tg mice compared with the non-Tg mice, which was verified by the histological evaluation that showed an increased, relatively large vessel. According to a previous microvascular MR study using stroke model, the microvascular network demonstrated changes within the ischemic penumbra in terms of microvessel density and average vessel size, referring to Q and VSI, respectively. In the initial infarct, or penumbra area, the reduced microvessel density (Q) and increased mean vessel radius were observed compared to the normal region. These observations could be explained by a more pronounced effect of edema on the compression of small 
capillaries as compared to larger arterioles and venules, leading to a shift in the calculated average vessel size. The vasodilation due to the autoregulation of the cerebral vascular network further results in the elevation of the VSI in the ischemic tissue. The VSI is able to monitor the dilation and contraction of microvessels [40; 41]. In $\mathrm{AD}$, susceptibility differences due to amyloid plaque causes magnetic field distortions in the vicinity of the blood vessels, resulting in a decrease of the transverse relaxation times of protons in the extracellular or extravascular space, and intravascular presence of amyloid plaque in the vessel wall. The dephasing mechanisms that may generate signal changes in GE and SE experiments are related to the maximum value of the magnetic field gradient induced at the surface of a vessel. Therefore, we expect the significance of increased VSI in Tg mice to be proportionate to the susceptibility difference. In addition, previous studies showed that VSI was correlated with pathologic diagnosis, and histologic estimates, taking into account the diffusion rate and CA concentration $[19 ; 26]$.

The microvessel density $(\mathrm{Q})$ is related to the VWF level, which is expected to increase capillary density in $\mathrm{AD}$. The accurate quantification of mean vessel density is dependent on diffusion values on tissue microstructure, different tissue types, and in pathologic conditions [28; 39]. Although a previous animal study showed decreased Q in Tg mice compared with non-Tg mice [42], our results showed no significant differences between the two groups of mice on ADC and Q. Amyloid plaque causes microvascular injury, in which the vessel radius may be reduced, and effects on Larmor frequency at the vessel surface. As a result, diffusion length is reduced. The $\mathrm{Q}$ is independent of the susceptibility difference or $\Delta \mathrm{R}_{2} *$ induced by the CA. The quantity Q parameter, determined from the shifts in the transverse relaxation rates induced by the CA, only depends on intrinsic tissue properties independent of the CA concentration, and is related to microvessel density [24; 31]. This may lead to a reduction in the underestimation of intrinsic blood paramagnetism. For estimation of $\mathrm{Q}$ between normal tissue and microvascular compromised tissue, the comparison is not fitted because the maximum signal drop in the microvascular compromised tissue is reached much later than in normal tissue, with a significantly lower concentration of CA $[31 ; 43]$. The concept of $\mathrm{Q}$ is referred to as the parameter, indicating the relative change of microvessel density, instead of quantitative microvessel density. For the validity of analytic expressions relating Q to the microvessel density, a high dose of CA is required [44]. Therefore, the physiological interpretation of this parameter could be much more complicated than VSI.

We used the $\mathrm{M}_{\mathrm{V}} \mathrm{WI}\left(\Delta \mathrm{R}_{2}{ }^{*} \times \Delta \mathrm{R}_{2}\right)$ as a voxel tool to map small blood vessels. It provides enhanced 
sensitivity to visualize intra-cortical penetrating arterioles and venules deep within the animal brain through multiplication of segmentation and quantification [22]. In this study, we found increased MvWI in the Tg mice compared with the non-Tg mice. We expected that the microvascular blood volume $\left(\Delta \mathrm{R}_{2}\right)$ would be increased in the $\mathrm{Tg}$ mice. Severe air-tissue interface blurring artifacts and overestimation of vessel radius due to $\Delta \mathrm{R}_{2} *$ under the magnetic field of high susceptibility was reduced by the effect of $\Delta \mathrm{R}_{2}$ below the spatial resolution. Thus, the sensitivity of small blood vessels was increased with the solution of an extravascular signal contribution caused by relatively large-sized vessels, arterioles and venules, by MvWI, especially for angiogenesis [45].

\section{Limitation}

In this study, we performed microvascular imaging using a 7-T animal MRI system in AD-model mice using blood pool CA of MION, with the advantage that the blood half-life of MION is several hours. However, in a human AD brain study, we cannot use MION; instead, we must use a Gadolinium-based contrast agent, as it is commonly applied in human studies. Most clinical studies in humans are performed using a 3-T MRI system. Therefore, to investigate microvascular changes in a human $\mathrm{AD}$ brain, we need to use 3-T MRI with a gadolinium-based contrast agent. This could be challenging for the study of microvascular imaging in human brain; however, it is important to evaluate microvascular alterations in the human AD brain.

\section{Conclusions}

We investigated cerebral microvascular injuries in the AD-model mouse brain using MRI techniques. We found that microvascular indices, including VSI, were significantly increased in the Tg mouse group compared with the non-Tg mouse group in the somatosensory cortex area, reflecting the vascular morphologic pathology of the Tg mice. This result may be related to vascular pathology or damages of the neurovascular unit in AD. Based on our results, investigation of microvascular structure in the human AD brain could be worthwhile using a clinical MRI system for early diagnosis and treatment monitoring in AD. 


\section{Declarations}

\section{- Ethics Approval and consent to participate}

All procedures performed in studies involving animals were in accordance with the ethical standards of the institution or practice at which the studies were conducted. The animal experiment was approved by the Institutional Animal Care and Use Committee of Ulsan National Institute of Science and Technology (UNISTIACUC-16-26).

\section{- Consent for publication}

Not applicable

\section{- Availability of data and materials}

The datasets used and/or analysed during the current study are available from the corresponding author on reasonable request.

\section{- Competing interests}

The authors declare that they have no competing interests.

\section{- Funding}

Ministry of Education (2016R1D1A1B03930720), Republic of Korea.

Ministry of Science and ICT (No. 2020R1A2C1004749, GHJ), Republic of Korea.

\section{- Author's contributions}

Each author has made following substantial contributions to the article. 
Suk-Ki Chang: have drafted the work.

JeongYeong Kim: acquisition, analysis of data.

DongKyu Lee: the creation of new software used in the work.

Chang Hyun Yoo: acquisition, analysis, or interpretation of data.

Seokha Jin: the creation of new software used in the work.

Hak Young Rhee: substantially revised it.

Chang-Woo Ryu: substantially revised it.

Jong Kil Lee: the creation of new software used in the work.

HyungJoon Cho: the creation of new software used in the work.

Geon-Ho Jahng: Corresponding author; conception or design of the work; substantially revised it.

\section{- Acknowledgements}

The authors thank Dr. Keigo Hikishima for helping to processing voxel-based analysis. This study was supported by the Basic Science Research Program through the National Research Foundation (NRF) of Korea funded by the Ministry of Education (2016R1D1A1B03930720) and the National Research Foundation of Korea (NRF) grant funded by Ministry of Science and ICT (No. 2020R1A2C1004749, GHJ), Republic of Korea.

\section{- Authors' information}

SK Chang ${ }^{1}, \quad$ cskclick@gmail.com

JY Kim ${ }^{2}, \quad$ rlawjddud73@gmail.com

DK Lee ${ }^{3}, \quad$ dangkyu00@unist.ac.kr

CH Yoo ${ }^{4}, \quad$ yewzzang92@gmail.com

SH Jin ${ }^{3}, \quad$ ssoek017@ naver.com 
HY Rhee ${ }^{5}, \quad$ azzo73@gmail.com

CW Ryu ${ }^{6}, \quad$ md.cwryu@gmail.com

JK Lee ${ }^{7}, \quad$ jklee3984@gmail.com

HJ Cho ${ }^{3}, \quad$ hjcho@unist.ac.kr

GH Jahng ${ }^{6^{*}} \quad$ ghjahng@gmail.com

${ }^{\mathbf{1}}$ Hallym University medical center, Hwasung, South Korea

${ }^{2}$ Department of Physics, Undergraduate School, Kyung Hee University, \#26 Kyungheedae-ro, Dongdaemun-gu, Seoul 02447, Korea

${ }^{3}$ Department of Biomedical Engineering, Ulsan National Institute of Science and Technology, Ulsan, South Korea

${ }^{4}$ Department of Physics, Graduate School, Kyung Hee University, \#26 Kyungheedae-ro, Dongdaemun-gu, Seoul 02447, Korea

${ }^{5}$ Department of Neurology, Kyung Hee University Hospital at Gangdong, College of Medicine, Kyung Hee University, \#892 Dongnam-ro, Gangdong-gu, Seoul 05278 Republic of Korea

${ }^{6}$ Department of Radiology, Kyung Hee University Hospital at Gangdong, College of Medicine, Kyung Hee University,\#892 Dongnam-ro, Gangdong-gu, Seoul 05278 Republic of Korea

${ }^{7}$ Department of Pharmacy, College of Pharmacy, Kyung Hee University, \#26 Kyungheedae-ro, Dongdaemun-gu, Seoul 02447, Korea

\section{References}

[1] Hunter JM, Kwan J, Malek-Ahmadi M, Maarouf CL, Kokjohn TA, Belden C, et al. Morphological and pathological evolution of the brain microcirculation in aging and Alzheimer's disease. PloS one 7(5): e36893.(2012).

[2] Postupna N, Keene CD, Crane PK, Gonzalez-Cuyar LF, Sonnen JA, Hewitt J, et al. Cerebral 
cortical Abeta42 and PHF-tau in 325 consecutive brain autopsies stratified by diagnosis, location, and APOE. Journal of neuropathology and experimental neurology 74(2): 100-9.(2015).

[3] Breteler MM. Vascular risk factors for Alzheimer's disease: an epidemiologic perspective. Neurobiology of aging 21(2): 153-60.(2000).

[4] Patel NS, Quadros A, Brem S, Wotoczek-Obadia M, Mathura VS, Laporte V, et al. Potent antiangiogenic motifs within the Alzheimer beta-amyloid peptide. Amyloid : the international journal of experimental and clinical investigation : the official journal of the International Society of Amyloidosis 15(1): 5-19.(2008).

[5] Paris D, Humphrey J, Quadros A, Patel N, Crescentini R, Crawford F, et al. Vasoactive effects of A beta in isolated human cerebrovessels and in a transgenic mouse model of Alzheimer's disease: role of inflammation. Neurological research 25(6): 642-51.(2003).

[6] Thal DR, Capetillo-Zarate E, Larionov S, Staufenbiel M, Zurbruegg S, Beckmann N. Capillary cerebral amyloid angiopathy is associated with vessel occlusion and cerebral blood flow disturbances. Neurobiology of aging 30(12): 1936-48.(2009).

[7] Herzig MC, Van Nostrand WE, Jucker M. Mechanism of cerebral beta-amyloid angiopathy: murine and cellular models. Brain pathology (Zurich, Switzerland) 16(1): 40-54.(2006).

[8] Altman R, Rutledge JC. The vascular contribution to Alzheimer's disease. Clin Sci (Lond) 119(10): 407-21.(2010). 
[9] Brown WR. A review of string vessels or collapsed, empty basement membrane tubes. Journal of Alzheimer's disease : JAD 21(3): 725-39.(2010).

[10] Drachman DA, Smith TW, Alkamachi B, Kane K. Microvascular changes in Down syndrome with Alzheimer's-type pathology: Insights into a potential vascular mechanism for Down syndrome and Alzheimer's disease. Alzheimer's \& dementia : the journal of the Alzheimer's Association 13(12): 1389-96.(2017).

[11] Lai AY, Dorr A, Thomason LA, Koletar MM, Sled JG, Stefanovic B, et al. Venular degeneration leads to vascular dysfunction in a transgenic model of Alzheimer's disease. Brain 138(Pt 4): 1046 58.(2015).

[12] Zenaro E, Piacentino G, Constantin G. The blood-brain barrier in Alzheimer's disease. Neurobiology of disease 107: 41-56.(2017).

[13] Weller RO, Djuanda E, Yow HY, Carare RO. Lymphatic drainage of the brain and the pathophysiology of neurological disease. Acta neuropathologica 117(1): 1-14.(2009).

[14] Iliff JJ, Wang M, Liao $Y$, Plogg BA, Peng W, Gundersen GA, et al. A paravascular pathway facilitates CSF flow through the brain parenchyma and the clearance of interstitial solutes, including amyloid beta. Science translational medicine 4(147): 147ra11.(2012).

[15] Guerreiro RJ, Gustafson DR, Hardy J. The genetic architecture of Alzheimer's disease: beyond APP, PSENs and APOE. Neurobiology of aging 33(3): 437-56.(2012).

[16] Salloway S, Gur T, Berzin T, Tavares R, Zipser B, Correia S, et al. Effect of APOE genotype on 
microvascular basement membrane in Alzheimer's disease. Journal of the neurological sciences 203-204: 183-7.(2002).

[17] Zipser BD, Johanson CE, Gonzalez L, Berzin TM, Tavares R, Hulette CM, et al. Microvascular injury and blood-brain barrier leakage in Alzheimer's disease. Neurobiology of aging 28(7): $977-$ 86.(2007).

[18] Tropres I, Grimault S, Vaeth A, Grillon E, Julien C, Payen JF, et al. Vessel size imaging. Magn Reson Med 45(3): 397-408.(2001).

[19] Tropres I, Pannetier N, Grand S, Lemasson B, Moisan A, Peoc'h M, et al. Imaging the microvessel caliber and density: Principles and applications of microvascular MRI. Magn Reson Med 73(1): 325-41.(2015).

[20] Oostendorp M, Post MJ, Backes WH. Vessel growth and function: depiction with contrastenhanced MR imaging. Radiology 251(2): 317-35.(2009).

[21] Dennie J, Mandeville JB, Boxerman JL, Packard SD, Rosen BR, Weisskoff RM. NMR imaging of changes in vascular morphology due to tumor angiogenesis. Magn Reson Med 40(6): 793-9.(1998). [22] Jung HS, Jin SH, Cho JH, Han SH, Lee DK, Cho H. UTE-DeltaR2 -DeltaR2 * combined MR whole-brain angiogram using dual-contrast superparamagnetic iron oxide nanoparticles. NMR Biomed 29(6): 690-701.(2016).

[23] Kleibeuker EA, Griffioen AW, Verheul HM, Slotman BJ, Thijssen VL. Combining angiogenesis inhibition and radiotherapy: a double-edged sword. Drug Resist Updat 15(3): 173-82.(2012). 
[24] Pannetier N, Lemasson B, Christen T, Tachrount M, Tropres I, Farion R, et al. Vessel size index measurements in a rat model of glioma: comparison of the dynamic (Gd) and steady-state (ironoxide) susceptibility contrast MRI approaches. NMR Biomed 25(2): 218-26.(2012).

[25] Sarraf M, Perles-Barbacaru AT, Nissou MF, van der Sanden B, Berger F, Lahrech H. RapidSteady-State-T1 signal modeling during contrast agent extravasation: toward tumor blood volume quantification without requiring the arterial input function. Magnetic resonance in medicine 73(3): 1005-14.(2015).

[26] Kang HY, Xiao HL, Chen JH, Tan Y, Chen X, Xie T, et al. Comparison of the Effect of Vessel Size Imaging and Cerebral Blood Volume Derived from Perfusion MR Imaging on Glioma Grading. AJNR American journal of neuroradiology 37(1): 51-7.(2016).

[27] Lugo-Hernandez E, Squire A, Hagemann N, Brenzel A, Sardari M, Schlechter J, et al. 3D visualization and quantification of microvessels in the whole ischemic mouse brain using solventbased clearing and light sheet microscopy. J Cereb Blood Flow Metab 37(10): 3355-67.(2017).

[28] Zerbi V, Jansen D, Dederen PJ, Veltien A, Hamans B, Liu Y, et al. Microvascular cerebral blood volume changes in aging $\mathrm{APP}(\mathrm{swe}) / \mathrm{PS} 1$ (dE9) AD mouse model: a voxel-wise approach. Brain structure \& function 218(5): 1085-98.(2013).

[29] Oakley H, Cole SL, Logan S, Maus E, Shao P, Craft J, et al. Intraneuronal beta-amyloid aggregates, neurodegeneration, and neuron loss in transgenic mice with five familial Alzheimer's disease mutations: potential factors in amyloid plaque formation. J Neurosci 26(40): 10129- 
40.(2006).

[30] Hikishima K, Komaki Y, Seki F, Ohnishi Y, Okano HJ, Okano H. In vivo microscopic voxel-based morphometry with a brain template to characterize strain-specific structures in the mouse brain. Sci Rep 7(1): 85.(2017).

[31] Jensen JH, Chandra R. MR imaging of microvasculature. Magn Reson Med 44(2): 22430.(2000).

[32] Wu EX, Tang $\mathrm{H}$, Jensen JH. High-resolution MR imaging of mouse brain microvasculature using the relaxation rate shift index Q. NMR Biomed 17(7): 507-12.(2004).

[33] Bai J, Trinh TL, Chuang KH, Qiu A. Atlas-based automatic mouse brain image segmentation revisited: model complexity vs. image registration. Magn Reson Imaging 30(6): 789-98.(2012).

[34] Sadowski M, Pankiewicz J, Scholtzova H, Li YS, Quartermain D, Duff K, et al. Links between the pathology of Alzheimer's disease and vascular dementia. Neurochem Res 29(6): 1257-66.(2004).

[35] Thomas T, Miners S, Love S. Post-mortem assessment of hypoperfusion of cerebral cortex in Alzheimer's disease and vascular dementia. Brain 138(Pt 4): 1059-69.(2015).

[36] Schneider JA, Arvanitakis Z, Leurgans SE, Bennett DA. The neuropathology of probable Alzheimer disease and mild cognitive impairment. Ann Neurol 66(2): 200-8.(2009).

[37] Brown WR, Thore CR. Review: cerebral microvascular pathology in ageing and neurodegeneration. Neuropathol Appl Neurobiol 37(1): 56-74.(2011).

[38] Kiselev VG, Strecker R, Ziyeh S, Speck O, Hennig J. Vessel size imaging in humans. Magn 
Reson Med 53(3): 553-63.(2005).

[39] Yanev P, Seevinck PR, Rudrapatna US, Bouts MJ, van der Toorn A, Gertz K, et al. Magnetic resonance imaging of local and remote vascular remodelling after experimental stroke. J Cereb Blood Flow Metab 37(8): 2768-79.(2017).

[40] Xu C, Schmidt WU, Galinovic I, Villringer K, Hotter B, Ostwaldt AC, et al. The potential of microvessel density in prediction of infarct growth: a two-month experimental study in vessel size imaging. Cerebrovasc Dis 33(4): 303-9.(2012).

[41] Lin CY, Chang C, Cheung WM, Lin MH, Chen JJ, Hsu CY, et al. Dynamic changes in vascular permeability, cerebral blood volume, vascular density, and size after transient focal cerebral ischemia in rats: evaluation with contrast-enhanced magnetic resonance imaging. J Cereb Blood Flow Metab 28(8): 1491-501.(2008).

[42] Silva JN, Polesskaya O, Wei HS, Rasheed IY, Chamberlain JM, Nishimura C, et al. Chronic central nervous system expression of HIV-1 Tat leads to accelerated rarefaction of neocortical capillaries and loss of red blood cell velocity heterogeneity. Microcirculation 21(7): 664-76.(2014).

[43] Xu C, Schmidt WU, Villringer K, Brunecker P, Kiselev V, Gall P, et al. Vessel size imaging reveals pathological changes of microvessel density and size in acute ischemia. J Cereb Blood Flow Metab 31(8): 1687-95.(2011).

[44] Jensen $\mathrm{JH}$, Lu H, Inglese M. Microvessel density estimation in the human brain by means of dynamic contrast-enhanced echo-planar imaging. Magn Reson Med 56(5): 1145-50.(2006). 
[45] Lin $\mathrm{CY}$, Lin $\mathrm{MH}$, Cheung WM, Lin TN, Chen JH, Chang C. In vivo cerebromicrovasculatural visualization using 3D DeltaR2-based microscopy of magnetic resonance angiography (3DDeltaR2mMRA). Neuroimage 45(3): 824-31.(2009). 


\section{Abbreviations}

2DTW1: two dimensional T1-weighted image

ADC: apparent diffusion coefficient

BVf: blood volume fraction

CA: contrast agent

CBV: cerebral blood volume

DG: dentate gyrus

DWI: diffusion-weighted image

FA: flip angle

FLASH: fast low angle shot magnetic resonance imaging

FOV: field-of-view

GE: gradient-echo

HP: hippocampus

MESE: multi-echo spin-echo

MEGE: multi-echo gradient-echo

MION: monocrystalline iron oxide nanoparticle

mVD: mean vessel diameter

MvWI: microvessel weighted image

nonTg mouse: control mouse

Q: mean vessel density

RARE: rapid acquisition with refocused echoes

ROI: region-of-interest

SE: spin-echo

SOM: somatosensory cortex

T1 FLASH: T1-weighted fast low angle shot

VBM: voxel-based-morphometry

VSI: vessel size index

TE: echo time

Tg mouse: transgenic mouse

TH: thalamus

TR: repetition time 


\section{Figure Legends}

Figure 1. Scan protocol and imaging acquisition sequences

$\mathrm{R} 2$ and R2* maps were calculated to map blood volume fraction (BVf), vessel size index (VSI), mean vessel diameter (mVD), mean vessel density (Q), and microvessel-weighted imaging (MvWI).

T1 FLASH: T1-weighted fast low angle shot (FLASH) to obtain brain anatomic information 2DT1W: 2D T1-weighted (T1W) imaging to obtain brain structure

DWI: diffusion-weighted imaging (DWI) to map apparent diffusion coefficient (ADC)

ME GE: multi-echo (ME) gradient-echo (GE) to map R2* relaxation rate before (pre) and after (post) injection of contrast agent

ME SE: multi-echo (ME) spin-echo (SE) to map R2 relaxation rate before (pre) and after (post) injection of contrast agent

Pre \& Post: imaging before (pre) and after (post) injection of contrast agent

$\mathrm{CE}$ : contrast enhancement with the monocrystalline iron oxide nanoparticle (MION)

Figure 2. Regions-of-interest (ROIs) defined by the mouse brain atlas (a) and the results of the voxelbased analyses (b)

(a)ROIs were defined by using the mouse brain atlas based on the wfu_pickatlas tool (http://fmri.wfubmc.edu/software/pickatlas) and Franklin and Paxinos atlas [33]. The defined ROIs were the right and left somatosensory cortexes, CA1, hippocampi, dentate gyri, and thalami.

(b)ROIs were defined by using the result from the voxel-based analyses with the $5 \mathrm{~mm}$ radius sphere at the center locations of the coordinate of the significant difference of the microvascular indices between the non- $\mathrm{Tg}$ and Tg groups. The detailed areas were also listed in Table 1 as: cerebral cortex general region (ROI1), somatosensory cortex (ROI2, ROI3, ROI4), and auditory cortex (ROI5).

Figure 3. Representative maps of $\Delta R 2$ and $\Delta R 2 *$ relaxation rates, $A D C$, and the microvascular indices obtained from the non-Tg (10 months) and Tg (2 months) mice

All maps were calculated after applying the Gaussian kernel of $0.25 \times 0.25 \times 1 \mathrm{~mm}^{3}$ to reduce the noise. The window level for each microvascular index was the same for non-Tg and $\mathrm{Tg}$ mice.

$\Delta \mathrm{R} 2\left(\mathrm{~s}^{-1}\right)$ and $\Delta \mathrm{R} 2 *\left(\mathrm{~s}^{-1}\right)$ are the differences of the relaxation rates before (pre) and after (post) injection of the contrast agent: $\Delta \mathrm{R} 2=$ postR $2-$ preR 2 and $\Delta \mathrm{R} 2 *=$ postR $2 *$ - preR $2 *$. 
ADC, apparent diffusion coefficient $\left(10^{-3} \mathrm{~mm}^{2} / \mathrm{s}\right)$; BVf, blood volume fraction $(\%)$; mVD, mean vessel diameter (unitless); Q, mean vessel density $\left(\mathrm{s}^{-1 / 3}\right)$; VSI, vessel size index $(\mu \mathrm{m})$; MvWI, microvessel-weighted image $\left(\mathrm{s}^{-2}\right)$

Figure 4. Result maps of the voxel-based comparisons between the non-Tg and Tg groups of $\Delta R 2$ (a), blood volume fraction (BVf) (b), and mean vessel diameter (mVD), vessel size index (VSI), and microvessel-weighted image (MvWI) (c)

Red color indicates increased microvascular indices in the Tg mouse group compared with in the non-Tg mouse group. There were no areas shown in greater indices in the non- $\mathrm{Tg}$ mouse group than in the $\mathrm{Tg}$ group.

The significant level was used with the $\mathrm{p}=0.001$ without correcting the multiple comparisons and with the threshold of 30 contiguous voxels.

Figure 5. Representative results of comparisons of the microvascular indices obtained from the right somatosensory cortex (a.ROI2) and the hippocampus (b.HP) listed in the Table 2

Note that the result in MvWI is scaled by $1 / 10$.

The vertical axis indicates the values of each index and therefore the unit is depended on the index.

The vertical solid bar indicates the standard deviation of the index within the group.

“*” indicates a significant difference of the index between the two groups.

Figure 6. Brain tissue histology to obtain vascular structures of the left somatosensory cortexes (6a) and around the thalamus and hippocampus (6b) for non-Tg mice and 5xFAD Tg mice

In the left somatosensory cortexes, the microvascular vessels were destroyed in the $\mathrm{Tg}$ mouse and the relatively large vessel is enlarged. In the thalamus and hippocampus, the microvascular structures of the Tg mouse were similar to those of the non-Tg mouse, but the entire volume of the vascular was increased in the $\mathrm{Tg}$ mouse because some of vessels were enlarged in the diameter. 


\section{Figures}

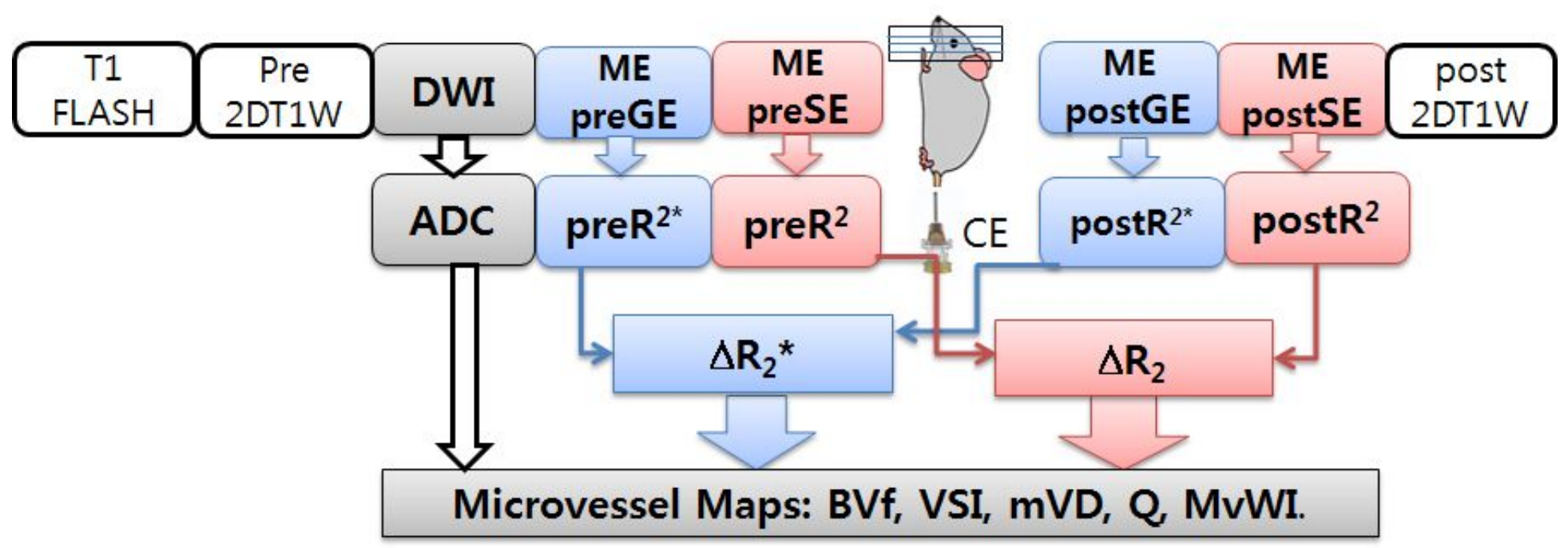

Figure 1

Scan protocol and imaging acquisition sequences R2 and R2* maps were calculated to map blood volume fraction (BVf), vessel size index (VSI), mean vessel diameter (mVD), mean vessel density $(\mathrm{Q})$, and microvessel-weighted imaging (MvWI). T1 FLASH: T1-weighted fast low angle shot (FLASH) to obtain brain anatomic information 2DT1W: 2D T1-weighted (T1W) imaging to obtain brain structure DWI: diffusion-weighted imaging (DWI) to map apparent diffusion coefficient (ADC) ME GE: multi-echo (ME) gradient-echo (GE) to map R2* relaxation rate before (pre) and after (post) injection of contrast agent ME SE: multi-echo (ME) spin-echo (SE) to map R2 relaxation rate before (pre) and after (post) injection of contrast agent Pre \& Post: imaging before (pre) and after (post) injection of contrast agent CE: contrast enhancement with the monocrystalline iron oxide nanoparticle (MION)

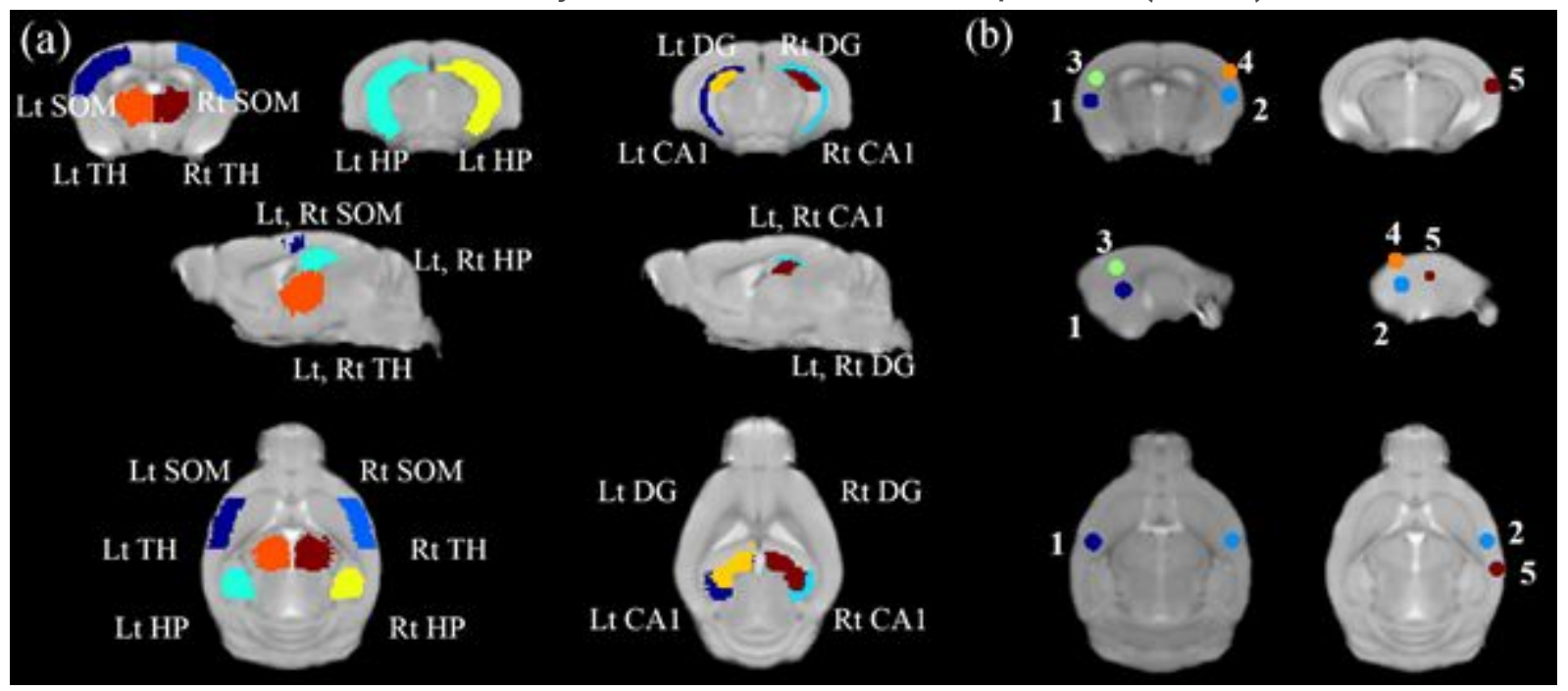

Figure 2 
Regions-of-interest (ROIs) defined by the mouse brain atlas (a) and the results of the voxel-based analyses (b) (a)ROIs were defined by using the mouse brain atlas based on the wfu_pickatlas tool (http://fmri.wfubmc.edu/software/pickatlas) and Franklin and Paxinos atlas [33]. The defined ROls were the right and left somatosensory cortexes, CA1, hippocampi, dentate gyri, and thalami. (b)ROls were defined by using the result from the voxel-based analyses with the $5 \mathrm{~mm}$ radius sphere at the center locations of the coordinate of the significant difference of the microvascular indices between the non- $\mathrm{Tg}$ and $\mathrm{Tg}$ groups. The detailed areas were also listed in Table 1 as: cerebral cortex general region (ROI1), somatosensory cortex (ROI2, ROI3, ROI4), and auditory cortex (ROI5).

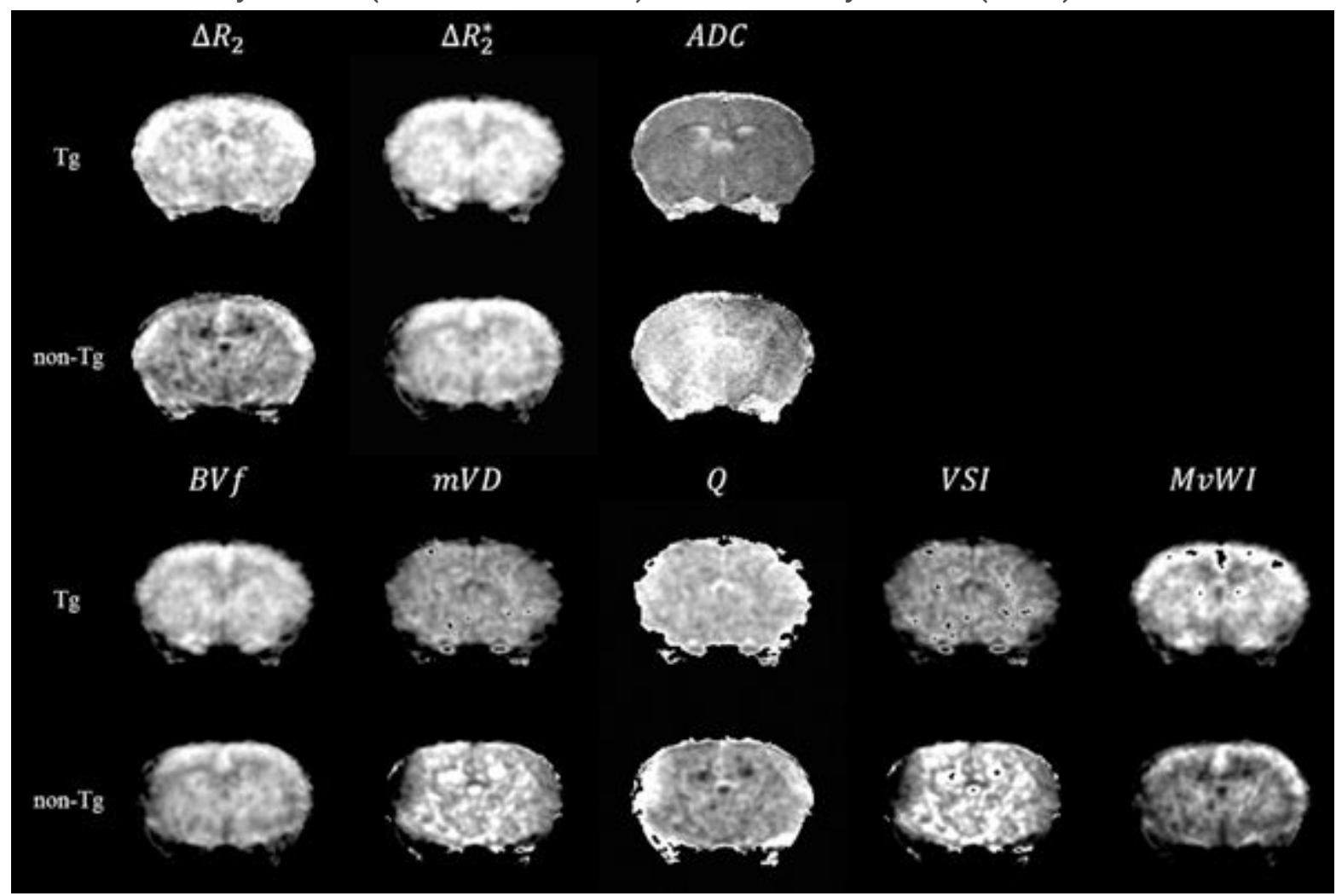

Figure 3

Representative maps of $\triangle R 2$ and $\triangle R 2 *$ relaxation rates, $A D C$, and the microvascular indices obtained from the non- $\mathrm{Tg}$ (10 months) and $\mathrm{Tg}$ ( 2 months) mice All maps were calculated after applying the Gaussian kernel of $0.25 \times 0.25 \times 1 \mathrm{~mm} 3$ to reduce the noise. The window level for each microvascular index was the same for non-Tg and Tg mice. $\Delta \mathrm{R} 2(\mathrm{~s}-1)$ and $\Delta \mathrm{R} 2{ }^{*}(\mathrm{~s}-1)$ are the differences of the relaxation rates before (pre) and after (post) injection of the contrast agent: $\triangle \mathrm{R} 2=$ postR2- preR2 and $\triangle R 2^{*}=$ postR2*- preR2* . ADC, apparent diffusion coefficient (10-3 mm2/s); BVf, blood volume fraction $(\%)$; mVD, mean vessel diameter (unitless); Q, mean vessel density ( $\mathrm{s}-1 / 3)$; VSI, vessel size index $(\mu \mathrm{m})$; MvWl, microvessel-weighted image ( $\mathrm{s}-2$ ) 

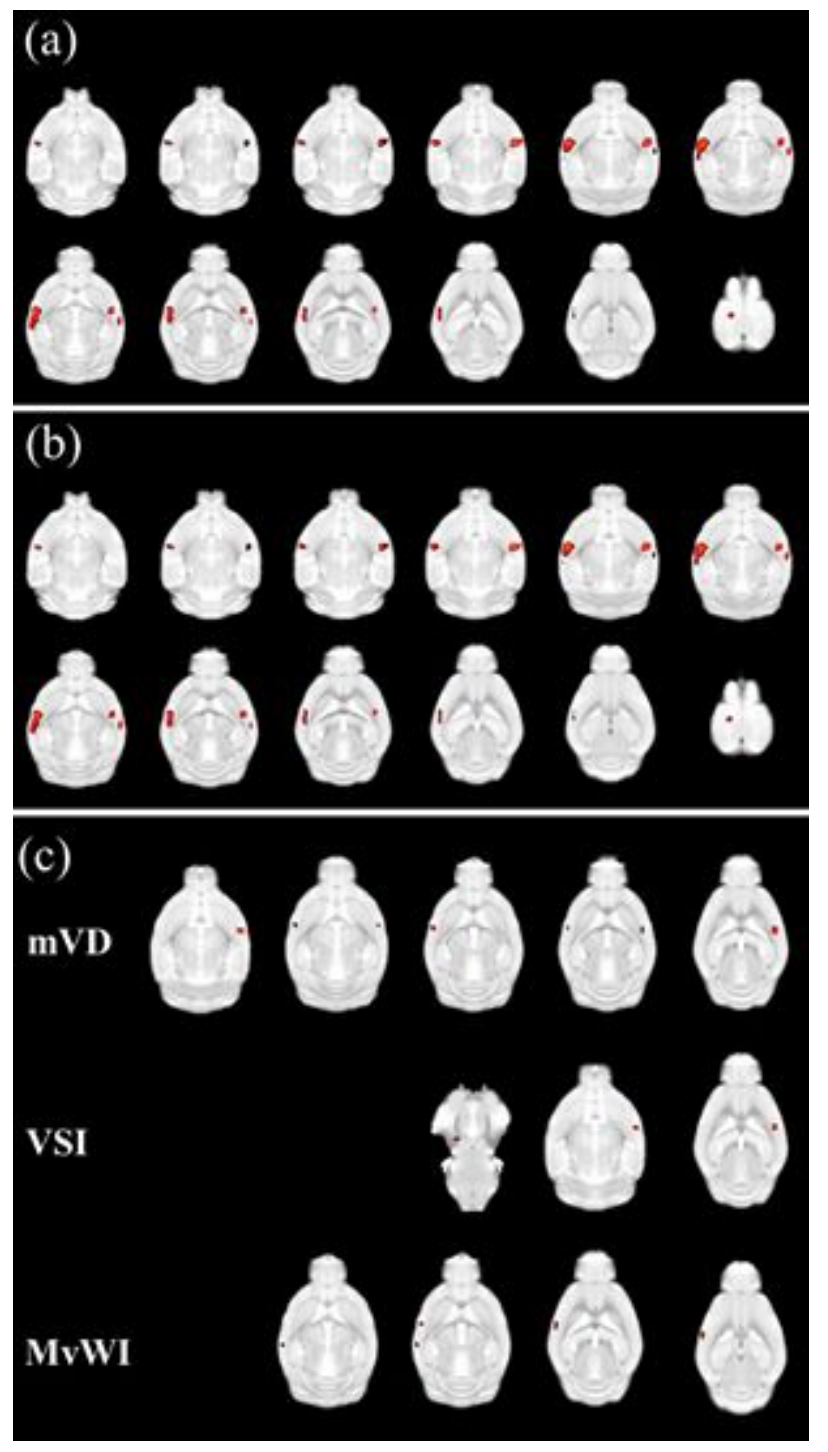

Figure 4

Result maps of the voxel-based comparisons between the non- $\mathrm{Tg}$ and $\mathrm{Tg}$ groups of $\Delta \mathrm{R} 2 *$ (a), blood volume fraction (BVf) (b), and mean vessel diameter (mVD), vessel size index (VSI), and microvesselweighted image (MvWI) (c) Red color indicates increased microvascular indices in the Tg mouse group compared with in the non-Tg mouse group. There were no areas shown in greater indices in the non- $\mathrm{Tg}$ mouse group than in the $\mathrm{Tg}$ group. The significant level was used with the $p=0.001$ without correcting the multiple comparisons and with the threshold of 30 contiguous voxels. 
(a)

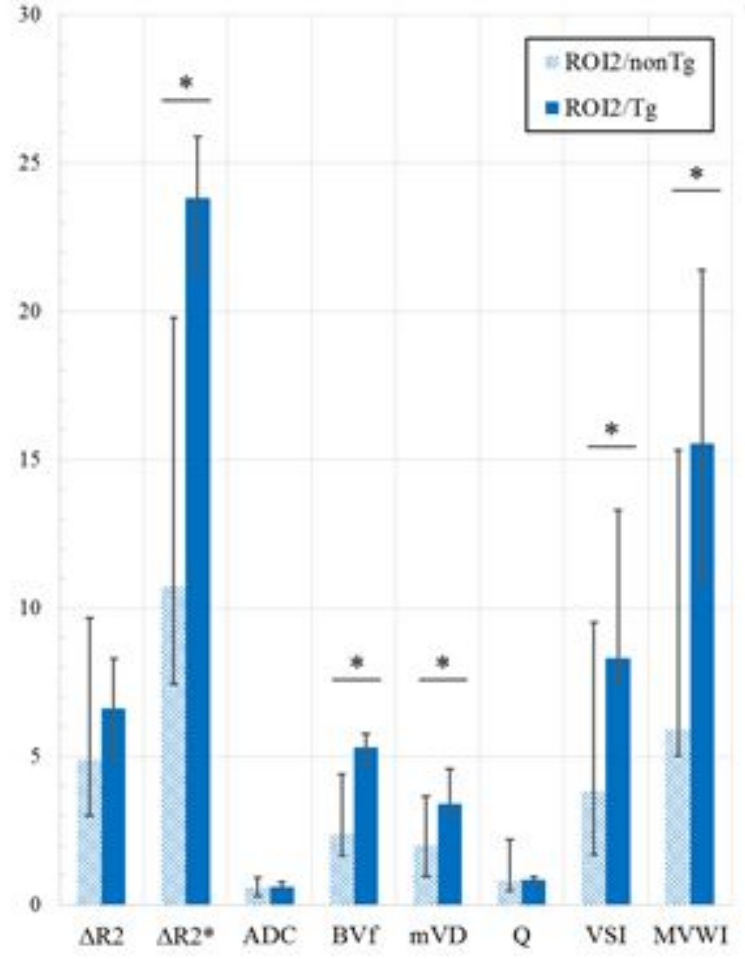

(b)

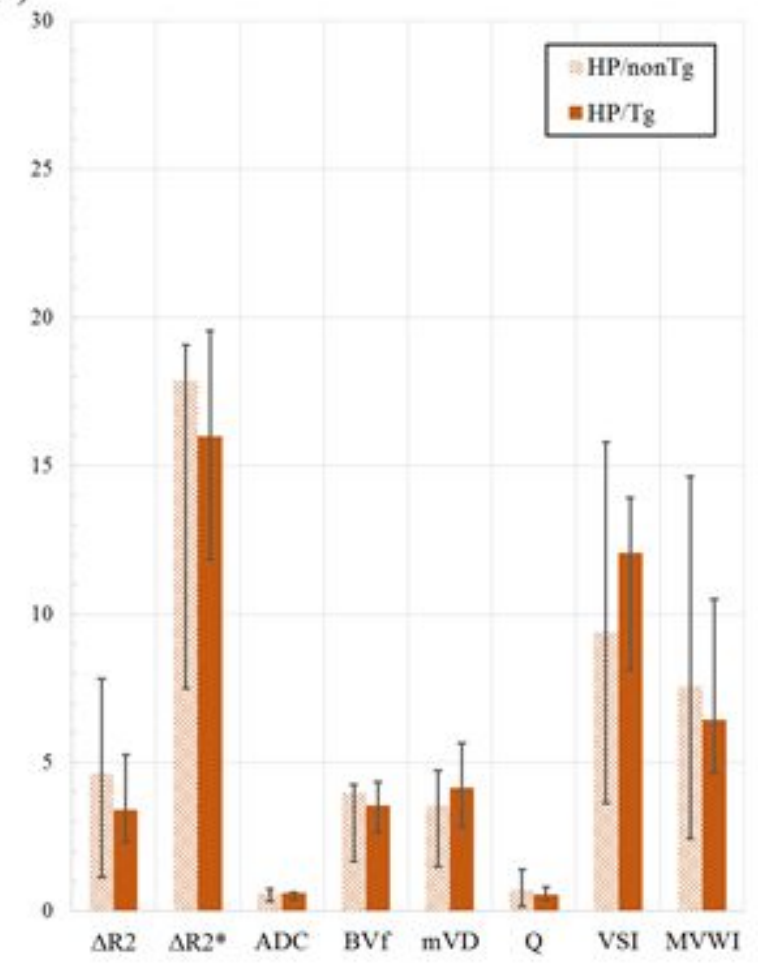

Figure 5

Representative results of comparisons of the microvascular indices obtained from the right somatosensory cortex (a.ROI2) and the hippocampus (b.HP) listed in the Table 2 Note that the result in MvWI is scaled by $1 / 10$. The vertical axis indicates the values of each index and therefore the unit is depended on the index. The vertical solid bar indicates the standard deviation of the index within the group. " $\star$ " indicates a significant difference of the index between the two groups. 


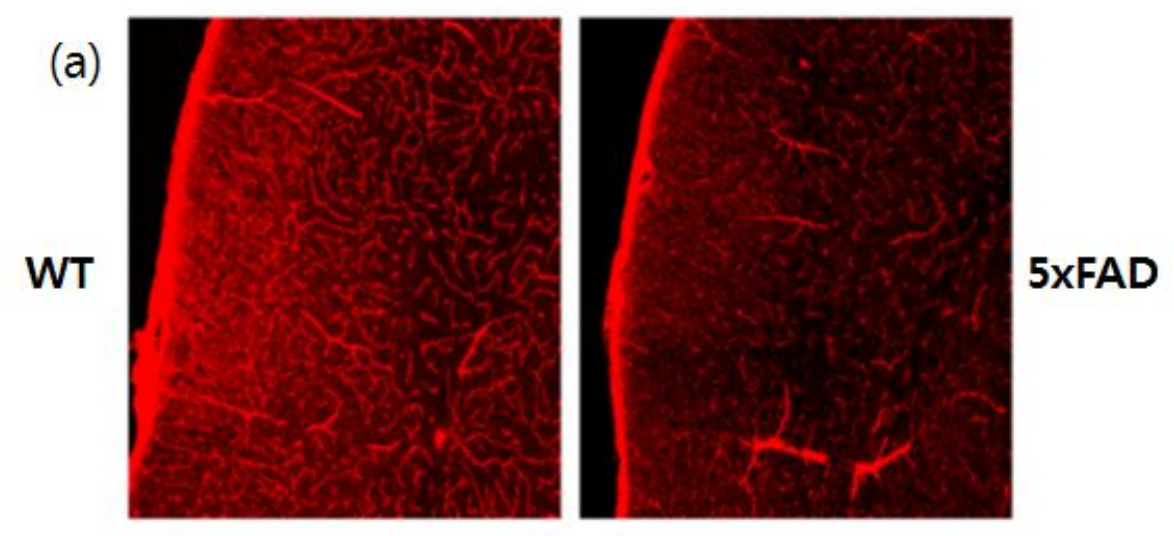

(b)
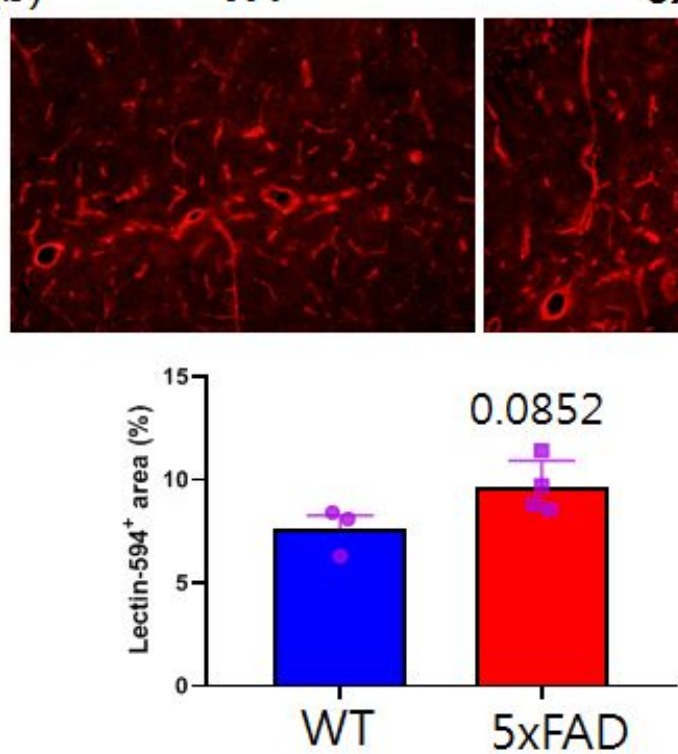

5XFAD
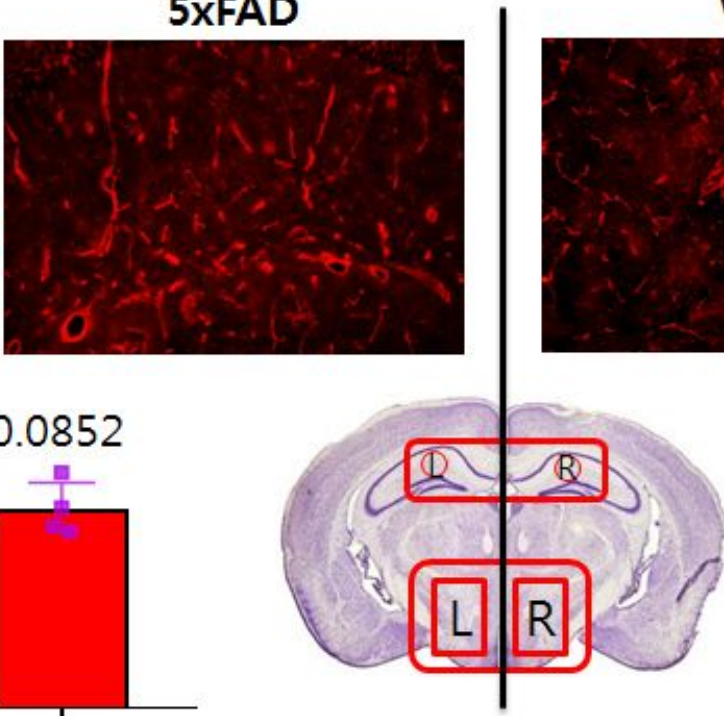

WT
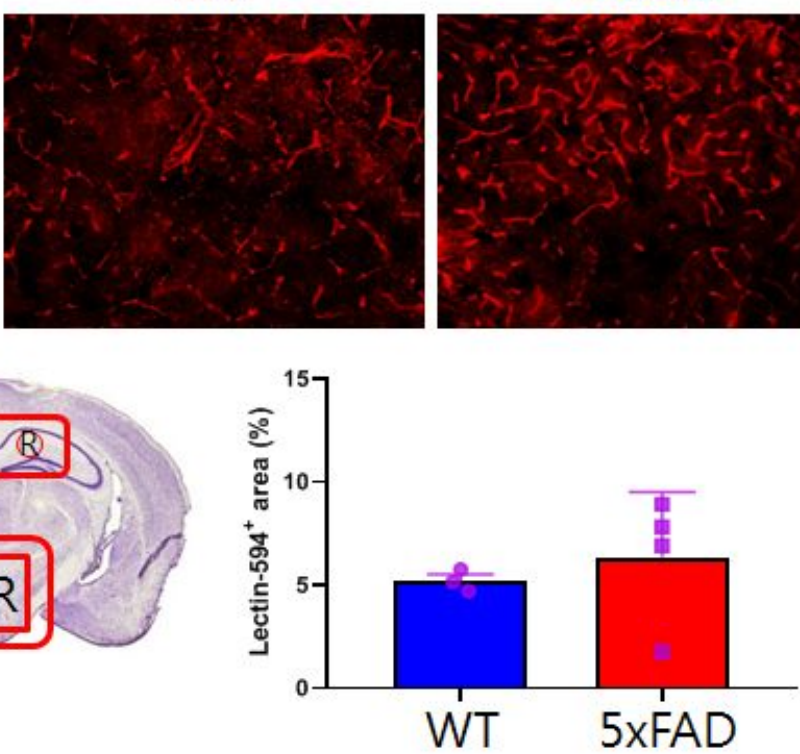

\section{Figure 6}

Brain tissue histology to obtain vascular structures of the left somatosensory cortexes (6a) and around the thalamus and hippocampus (6b) for non-Tg mice and 5xFAD Tg mice In the left somatosensory cortexes, the microvascular vessels were destroyed in the $\mathrm{Tg}$ mouse and the relatively large vessel is enlarged. In the thalamus and hippocampus, the microvascular structures of the Tg mouse were similar to those of the non-Tg mouse, but the entire volume of the vascular was increased in the Tg mouse because some of vessels were enlarged in the diameter. 